\title{
Les médecins ne sont pas des cibles
}

\section{Thomas Nierle}

Dr med., président de Médecins Sans Frontières Suisse

Imaginez quelques instants: vous êtes en train de travailler à l'hôpital, en pleine consultation. Soudain, vous entendez un sifflement strident, suivi d'une explosion assourdissante. Les murs tremblent, les vitres volent en éclats et des objets sont propulsés de toutes parts dans la pièce. Vous vous précipitez dehors pour comprendre ce qui s'est passé: terrifié, stupéfait, mais aussi par instinct parce que vous savez qu'il faut agir au plus vite. La scène que vous découvrez est effroyable: le service des urgences est en feu, en ruines. Il vient d'être bombardé. Vous entendez des gémissements, devinez des morceaux de membres çà et là, du sang partout. Certaines des victimes sont des gens que vous connaissez bien: vos patients, mais aussi des collaborateurs et des amis. Le chaos est indescriptible, insoutenable, mais vous n'avez pas le choix; votre rôle reste de soigner ceux qui peuvent encore l'être.

Plus d'un lecteur ou d'une lectrice se demanderont où je veux en venir en dépeignant une situation aussi déconnectée de la réalité. Pourtant, pour les médecins qui exercent en Syrie, au Yémen ou au Sud Soudan, ce scénario d'horreur est bel et bien la réalité. Les hôpi- taux de Kunduz, d'Alep et plus récemment celui d'Abs, au Yémen, ne sont que quelques-uns des établissements médicaux dirigés ou soutenus par Médecins Sans Frontières qui ont été bombardés et détruits au cours de l'année écoulée. Les patients, mais aussi les médecins, infirmiers et aides-soignants qui y ont perdu la vie sont innombrables.

Les bombardements aériens ne sont cependant que la partie la plus visible de la violence à laquelle font face actuellement les hôpitaux et leur personnel dans les zones de guerre. Assassinats de patients hospitalisés, tirs, pillages et menaces de mort dans les structures médicales ne cessent de réduire la capacité à soigner. Ces actes minent aussi peu à peu ce qu'il reste d'humanité dans les contextes de guerre et de violence.

\section{Les populations civiles, premières victimes}

Ce sont en fin de compte toujours les civils qui sont les plus durement touchés par cette brutalité. Ce sont des centaines de milliers de personnes qui, du jour au len-

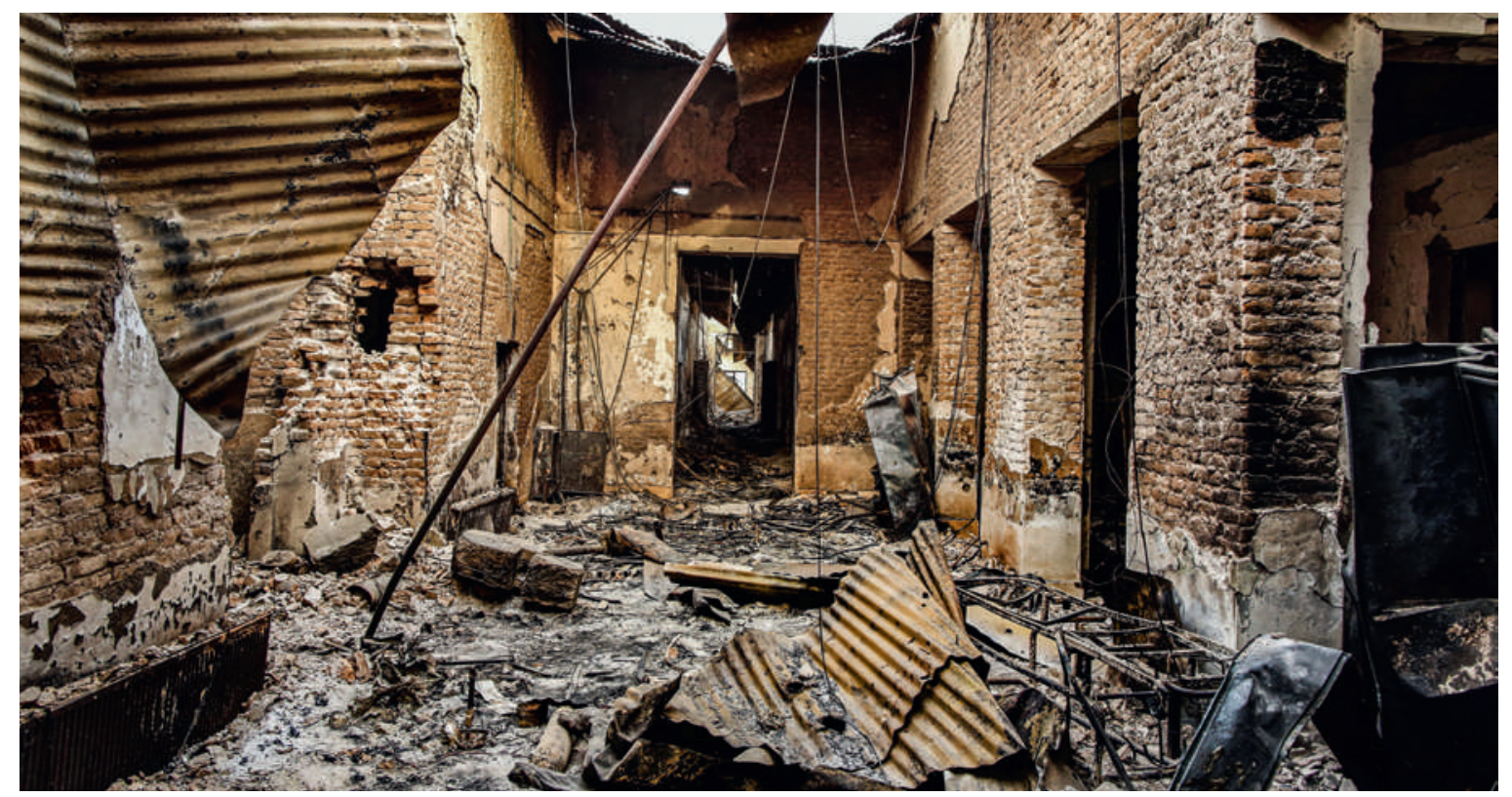

Le centre de traumatologie de MSF à Kunduz, en Afghanistan, a été la cible d’attaques aériennes. Celles-ci ont détruit I’hôpital et tué 42 personnes, dont 14 membres du personnel de MSF. 
demain à la suite d'une attaque, perdent tout accès à des soins médicaux lorsque qu'un hôpital doit fermer ses portes. Ce sont elles aussi qui meurent des suites de maladies faciles à traiter en temps normal, elles qui restent mutilés à vie car leurs blessures n’ont pas pu être soignées à temps.

L'hôpital est et doit rester un lieu de soin, d'humanité et d'espoir. Il semble aujourd'hui que réduire à néant cet ultime sanctuaire soit devenu une tactique de guerre. Le droit humanitaire international établit que les établissements de soins de santé doivent être protégés en temps de guerre et que ni les patients, ni le personnel médical ne peuvent être attaqués - encore moins assassinés. Mais avec la multiplication des guerres dites de contreterrorisme, l'application de ces règles juridiques est radicalement compromise. Qualifier son adversaire de "groupe terroriste» justifie le recours à n'importe quel moyen tant qu'il s'agit de lui infliger un maximum de pertes. Empêcher les combattants blessés d'accéder aux soins, terroriser la population civile jusqu'à ce qu'elle n'ait plus d'autre choix que l'exil... voilà notamment ce que à quoi peut condamner cette désignation. La terreur répond à la terreur, générant une spirale de brutalité où l'humanité est sacrifiée à l'autel de la guerre totale. L'impartialité, une valeur fondamentale de l'éthique médicale, devient une cible en soi, dans la mesure où soigner des combattants blessés équivaut à se ranger du côté de l'ennemi.

\section{La solidarité, moyen ultime}

Bien que la communauté internationale ait rappelé en mai dernier, dans une résolution de l'ONU, l'interdiction d'attaquer les établissements de soins et le personnel médical et humanitaire, cette initiative fait figure de "vœu pieux». Tant en Syrie qu'au Yémen, les attaques sont menées avec l'appui des grandes puissances mondiales. Qu'il s'agisse de livraisons d'armes, de soutien logistique ou de participation directe au conflit, la Russie, les Etats-Unis et la Grande-Bretagne portent une part de responsabilité dans ces attaques. Alors que les belligérants parlent de dégâts collatéraux lorsque des structures médicales sont bombardées, peut-on encore réellement considérer comme des accidents les 96 hôpitaux soutenus par MSF touchés par des bombes en Syrie en 2015? Ne s'agit-il pas plutôt de destruction systématique d'infrastructures civiles dont l'unique vocation est d'assurer les soins médicaux à la population?

On me demande souvent pourquoi nous nous acharnons encore à vouloir travailler dans ces régions. Ne devrions-nous pas plutôt nous retirer de ces zones, pour protéger nos collaborateurs? Il nous arrive en

\section{\#NotATarget - Evènements MSF}

Médecins Sans Frontières (MSF) visitera cet automne de nombreux hôpitaux suisses, dans le cadre de son initiative "\#NotA Target» («Pas une Cible»). Une exposition sera montrée et des soirées d'information à propos des attaques contre la mission médicale humanitaire seront organisées, incluant une projection de film et une discussion avec des membres de MSF.

Hôpitaux Universitaires de Genève Exposition du 3 au 9 octobre 2016

Soirée de commémoration le 3 octobre à partir de 18 h30 1 an après la tragédie de Kunduz

Centre hospitalier universitaire vaudois Exposition du 17 au 23 octobre 2016 Soirée thématique le 20 octobre à 17 h00

Hôpital universitaire de Berne

Exposition du 17 au 23 octobre 2016

Soirée thématique le 18 octobre à 18 h00

Hôpital Neuchâtelois

Exposition du 24 au 30 octobre 2016

Hôpital cantonal de Fribourg

Exposition du 24 au 30 octobre 2016

Soirée thématique le 26 octobre à $18 \mathrm{~h} 00$

Hôpital Universitaire de Bâle

Exposition du 31 octobre au 6 novembre 2016

Soirée thématique le $1^{\text {er }}$ novembre à $18 \mathrm{~h} 00$

Institut Tropical de Bâle

Exposition du 7 au 13 novembre 2016

Soirée thématique le $1^{\text {er }}$ novembre à 18 h00

Hôpital cantonal de Winterthour

Soirée thématique le 9 novembre à 18 h00

Hôpital Universitaire de Zurich

Exposition du 14 au 20 novembre 2016

Soirée thématique le 16 novembre à 18 h00

Plus d'informations sur www.msf.ch/event

effet d'être contraints d'évacuer des équipes d'endroits où leur sécurité n'est pas garantie, ou lorsque les incidents sont banalisés. Des membres du personnel médical restent toutefois sur place. Où la médecine peut apaiser les souffrances humaines, il y aura toujours des médecins et des soignants. Que Médecins Sans Frontières reste ou non dans un endroit ou un autre ne change rien à la nécessité de mettre un terme aux attaques sur les centres de soins. Les médecins qui exercent en zone de guerre sont tous nos collègues, et nous leur sommes solidaires. Comment pourrait-on faire autrement puisque nous sommes convaincus que chaque personne a le droit à la protection et à l'aide médicale, même en temps de guerre, même en temps de crise? Il est tout simplement intolérable que les médecins soient pris pour cibles!

Crédit photo

(c) Andrew Quilty 